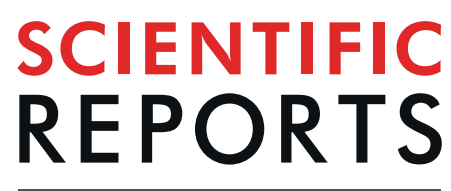

\title{
OPEN Prevalence of atrophic gastritis in southwest China and predictive strength of serum gastrin-17: A cross-sectional study (SIGES)
}

\begin{abstract}
Rui Wang ${ }^{1}$ \& Xin-Zu Chen $\mathbb{D}^{2,3,4^{*}}$
A hospital-based cross-sectional study in SIGES project was conducted during 2016.5-2017.5 in West China Hospital. It was aimed to observe the prevalence of atrophic gastritis (AG) in southwest China, and assess the diagnostic strength of serum gastrin-17 (G-17) in predicting AG in Chinese population. Asymptomatic healthy controls from health check-up, cancer-free patients with unspecific upper gastrointestinal symptoms, and histologically proven gastric cancer patients were eligible, if serum pepsinogen-I (PG-I), PG-II, and G-17 were detected. AG status was classified by the accredited cutoffs of PG-I ( $<70 \mathrm{ug} / \mathrm{L})$ and PG-I/II ratio $(<3)$. Totally, healthy controls $(n=9,425)$, symptomatic patients $(n=671)$ and gastric cancer patients $(n=305)$ were simultaneously observed, in which the prevalence of AG in southwest China were estimated as 15.9/1,000, 28.3/1,000, and 55.7/1,000 persons, respectively. The age-specific prevalence of $A G$ in healthy controls showed a significantly uphill trend ( $p$ for trend $<0.001$ ). Higher level of serum G-17 was significantly associated with increased risk of AG in healthy population $(15-30 \mathrm{pmol} / \mathrm{L}, \mathrm{aOR}=20.67,95 \% \mathrm{Cl} 9.17-46.55 ;>30 \mathrm{pmol} / \mathrm{L}, \mathrm{aOR}=314.41,95 \%$ $\mathrm{Cl}$ 166.10-595.12). Throughout the progression of stomach diseases, the diagnostic strength of serum G-17 for AG showed a downhill trend across more advanced situations. In despite of that, serum G-17 displayed a good performance in predicting $A G$ in the entire cross-sectional population (AUC $=0.92$, 95\% Cl 0.89-0.94; SEN = 85.5\%; SPE = 93.2\%; LR+=12.55; LR- =0.11). Population in southwest China had intermediate prevalence of $A G$, while the prevalence was increased over age or disease progression. High level of serum G-17 might be a reliable non-invasive measurement to predict AG in southwest Chinese population.
\end{abstract}

Atrophic gastritis is a well-established precursor of intestinal-type gastric cancer. The population prevalence of atrophic gastritis generally ranged from $2.1 \%$ to $8.2 \%^{1}$. The presence of atrophic gastritis was associated with the increased risk of gastric cancer during longitudinal observation in both the western and eastern populations ${ }^{2-4}$. Therefore, the China Consensus on Early Gastric Cancer Screening, Endoscopic Diagnosis and Treatment (2014) recommended to define patients with atrophic gastritis as a kind of high-risk candidates for gastric cancer screening $^{5}$. The European Helicobacter Study Group agreed on the statement "Serologic screening is suitable for clinical use in countries with a relatively low incidence of gastric cancer, because it enables endoscopic follow-up of caseswith an abnormal serologic profile suggesting atrophic gastritis" ${ }^{6}$. However, nationwide massive screening programs for gastric cancer were established only in Japan and Korea, but not in China and other nations yet ${ }^{7,8}$. Regarding the great health burden from gastric cancer in China ${ }^{9,10}$, particularly the relatively low proportion of early gastric cancer ${ }^{7,11}$, it would be necessary to investigate the relevant screening strategy covering precancerous lesions based on Chinese population.

Serum pepsinogen was widely used in clinical practice as biomarkers of stomach inflammation and mucosal lesions, including atrophic changes ${ }^{12,13}$. Serologic diagnosis of atrophic gastritis by combination of pepsinogen-I (PG-I) and PG-I/II ratio demonstrated excellent performance as a preferable non-invasive measurement ${ }^{14-16}$.

\footnotetext{
${ }^{1}$ Department of Gastroenterology, Nursing Section, West China Hospital, Sichuan University, Chengdu, China. ${ }^{2}$ Department of Gastrointestinal Surgery \& Laboratory of Gastric Cancer, West China Hospital, Sichuan University, Chengdu, China. ${ }^{3}$ Department of Gastrointestinal and Hernia Surgery, The Second People's Hosopital ofYibin $\bullet$ West China Yibin Hospital, Sichuan University, Yibin, China. ${ }^{4}$ Department of General Surgery, The First People's Hospital of Longquanyi • West China Longquan Hospital, Sichuan University, Chengdu, China. *email: chenxinzu@scu.edu.cn
} 


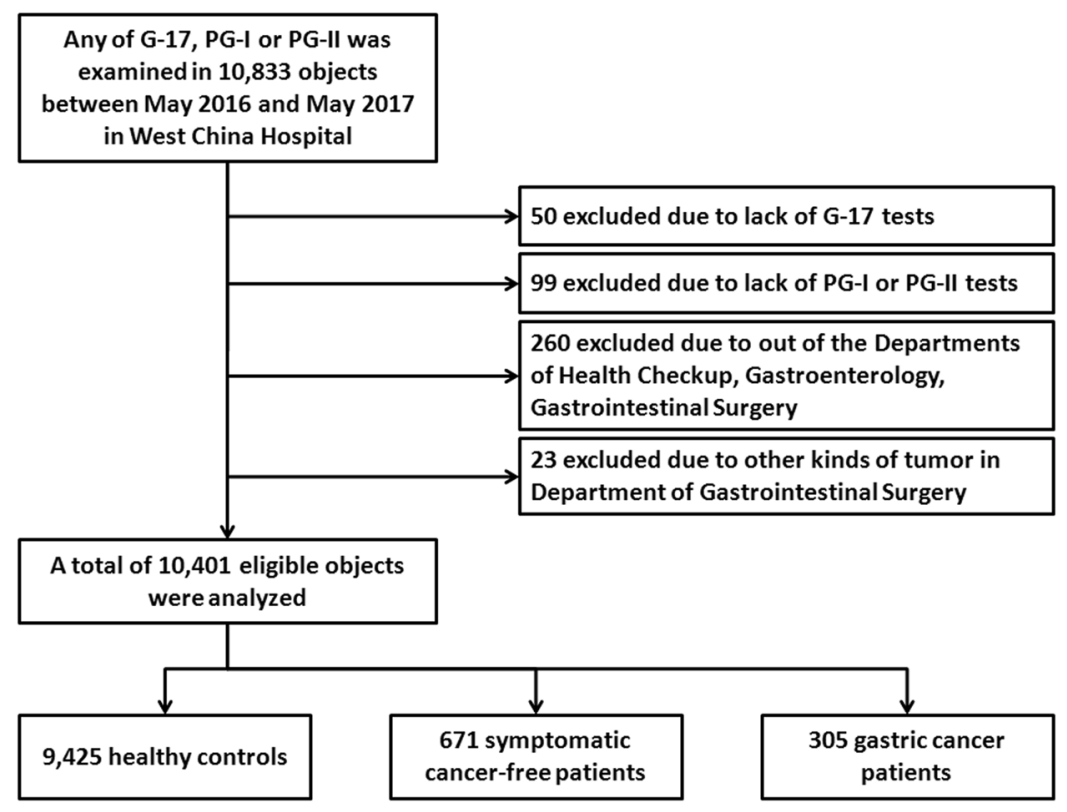

Figure 1. The flow chart of the SIGES cross-sectional study.

Particularly, pepsinogens was recommended as useful biomarkers to reflect gastric atrophy and identify high-risk subpopulation for gastric cancer by an Asia-Pacific consensus ${ }^{17}$. Additionally, gastrin-17 (G-17) was known as another non-invasive biomarker for atrophic gastritis ${ }^{18-20}$. G-17, a predominant form of antral hormone gastrins in plasma or tissue in antral mucosa, can regulate gastric acid secretion and growth of the gastric mucosa ${ }^{18,21}$. G-17 is almost exclusively produced by the antral G-cells, which can reflect the progression and severity of gastric mucosal diseases. Previous observational studies showed diverse results, and a meta-analysis found the diagnostic strength of G-17 for predicting atrophic gastritis was only $48 \%$ sensitivity and $79 \%$ specificity ${ }^{22}$. However, the relevant studies based on Chinese population were still sparse, and the extrapolation of serum G-17 test in Chinese massive screening requires further investigations.

Therefore, the SIGES project was aimed to investigate the prevalence of atrophic gastritis in southwest China, and simultaneously assess the diagnostic strength of serum G-17 in predicting atrophic gastritis in healthy persons, symptomatic cancer-free patients, and gastric cancer patients.

\section{Methods}

Study design. This was a retrospective hospital-based cross-sectional study, with the major mission of Sichuan Gastric Cancer Early Detection and Screening (SIGES) project. It was conducted in West China Hospital, Sichuan University, a central high-volume teaching hospital at Sichuan province in the southwest of China. The period of the study was between May 2016 and May 2017. The subjects covered healthy controls, symptomatic cancer-free patients, and gastric cancer patients, who were managed or treated in West China Hospital. A total of 10,401 observations were included in this SIGES study (Fig. 1).

Ethics. This hospital-based cross-sectional study retrospectively collected the participants' basic information and results of serum G-17, PG-I, and PG-II. The SIGES study was approved by the Biomedical Ethical Committee of West China Hospital, Sichuan University (id: 2015-151-V2). The informed consent was waived by the approval of the Biomedical Ethical Committee for the sake of retrospective nature, but the personal information was anonymised, when analyzing and reporting data.

Eligibility. The eligibility was reviewed and assessed thoroughly based on the electronic medical records reportedby general practioners, gastroenterologists, or gastrointestinal surgical oncologists: (1) The healthy controls were collected from the health checkup in the Center of Health Checkup. The general practitioners recorded them as the asymptomatic status and cancer-free status; (2) The symptomatic cancer-free patients were collected among the outpatients or inpatients from the Department of Gastroenterology and the Department of Gastrointestinal Surgery. These patients were diagnosed of chronic gastritis or functional dyspepsia due to unspecific upper gastrointestinal symptoms, and were recorded as cancer-free status by gastroenterologists or gastrointestinal surgical oncologists; (3) The gastric cancer patients were collected from the Department of Gastrointestinal Surgery. The diagnosis was proved by endoscopic biopsy and pathology, regardless of stage. Other kinds of malignancies were excluded, such as lymphoma and gastrointestinal stromal tumor. In addition, all the included observations should be tested the serum G-17, PG-I, and PG-II together. For the gastric cancer patients, the serologic tests should be performed preoperatively, while the postoperative tests would not be considered. 
Serology. Fasting blood samples were obtained from all observations at health checkup, outpatient visit, or surgical ward. The fresh sample was transferred to the Department of Clinical Laboratory in time and the serum was tested in the same day. The serologic tests of G-17, PG-I, and PG-II were in-house determined by enzyme-linked immunosorbent assay (ELISA; BioHit) according to the manufacturer's instructions. The in-house cutoffs of normal references were, PG-I 70-165 ug/L, PG-II 3-15 ug/L, PG-I/II ratio 7-20, and G-17 1-15 pmol/L, respectively. The atrophic gastritis was defined as PG-I $<70.0 \mathrm{ug} / \mathrm{L}$ and PG-I/II ratio $<3.0$, which was a comprehensively accepted cutoff for both the western and eastern populations ${ }^{3,13,23,24}$. Among those, if PG-I $<20.0$ $\mathrm{ug} / \mathrm{L}$ and PG-I/II ratio $<3.0$, they were defined as severe atrophy, while the others were mild-moderate atrophy.

Statistics. Demographic characteristics and serologic results of the observations were compared by Chi-square test for categorical variables, and Wilcoxon rank-sum test for ranked variables or continuous variables without normality distribution. Shapiro-Wilk test for normal data was used to check the normality of variables. Provided multi-group comparison, the Kruskal-Wallis rank test was performed initially. Spearman rho correlation test was used analyzed the association of G-17 with PG-I, PG-II, and PG-I/II ratio. The correlation coefficients were estimated, and relationship was classified by the absolute value of coefficients, as strong (0.7-1), moderate (0.5-0.7), weak (0.3-0.5), and none (0-0.3). The non-parametric trend test was used to test the changes of prevalence over age groups. Multivariate analyses were performed by Logistic regression, and adjusted odds ratio (aORs) with 95\% confidence intervals (CIs) were estimated. Non-parametric ROC analysis without covariates was used to assess the capability of predicting atrophic gastritis status, while sensitivity (SEN), specificity (SPE), accurancy (ACC), positive likelihood ratio (LR+), and negative likelihood ratio (LR-) were additionally estimated. The $\mathrm{LR}+>10.0$ or $\mathrm{LR}-<0.1$ presented a strong diagnostic power. The area under the curve (AUC) with standard error (SE) was calculated. The cutoffs of serum G-17 $>1 \mathrm{pmol} / \mathrm{L},>15 \mathrm{pmol} / \mathrm{L}$, and $>30 \mathrm{pmol} / \mathrm{L}$ were applied while estimating AUCs. Additionally, the maximal Youden index $(=\mathrm{SEN}+\mathrm{SPE})$ was calculated to determine the optimal cutoff of serum G-17. The strength of AUCs was classified as mild (0.5-0.7), moderate (0.7-0.9), and strong (0.9-1). The difference of AUCs were compared by the $\mathrm{Z}$ test. All statistical tests were two-sided and statistical significance was defined as $\mathrm{p}<0.05$. The STATA/SE 12.0 software was used for statistical analysis.

\section{Results}

In the SIGES study, 9,425 healthy controls, 671 symptomatic cancer-free patients, and 305 gastric cancer patients were included. The demography and results of serum PG-I, PG-II, PG-I/II ratio, and G-17 were displayed in the Table 1 and Supplementary Fig. 1. There was only weak-moderate correlation of serum G-17 with serum PG-I, PG-II, or PG-I/II ratio (Supplementary Fig. 2).

The prevalence of atrophic gastritis were 15.9 (95\% CI 13.4-18.4) per 1,000 persons in healthy population, 28.3 (95\% CI 15.8-40.9) per 1,000 persons in upper GI symptomatic patients, and 55.7 (95\% CI 30.0-81.5) per 1,000 persons in gastric cancer patients, respectively (Fig. 2 ). In the healthy controls, the age-specific prevalence of atrophic gastritis showed a significantly uphill trend ( $\mathrm{p}$ for trend $<0.001$ ) (Fig. 2). The prevalence were $0,5.1$ (95\% CI 2.2-7.9), 14.5 (95\% CI 11.4-17.6), 41.3 (95\% CI 30.1-52.5), and 97.6 (95\% CI 6.7-188.4) per 1,000 persons in $<20$ years, $20-39$ years, $40-59$ years, $60-79$ years, and $\geq 80$ years age groups, respectively.

Sex was not associated with the risk of atrophic gastritis (Table 2). Concerning age, only $\geq 60$ years was an independent risk factor in healthy population ( $\mathrm{aOR}=3.02,95 \% \mathrm{CI} 1.46-6.21$ ). The serum $\mathrm{G}-17$ level presented uphill trends over the severity of atrophic gastritis in different subjects (all p for trend $<0.05)$ (Fig. 3). Additionally, in healthy controls, high level $(\mathrm{aOR}=20.67,95 \% \mathrm{CI} 9.17-46.55)$ and very high level $(\mathrm{aOR}=314.41$, 95\% CI 166.10-595.12) of serum G-17 were associated with the increased risks of atrophic gastritis. However, low level of serum G-17 was not associated with the risk of atrophic gastritis (Table 2), and it was the reason why low and normal levels of serum G-17 were combined as one subgroup in the present study. Moreover, throughout the progression of stomach diseases, only very high level of serum G-17 was always associated with the increased risk of atrophic gastritis in symptomatic cancer-free patients $(\mathrm{aOR}=42.40,95 \% \mathrm{CI} 11.63-154.66)$ and gastric cancer patients $(\mathrm{aOR}=5.50,95 \% \mathrm{CI} 1.78-17.02)$, respectively.

Regarding none inverse risk of atrophic gastritis between low and normal levels of serum G-17, the optimal cutoff of prediction would be reasonable. The in-house upper limit of serum G-17 ( $\leq 15 \mathrm{pmol} / \mathrm{L})$ demonstrated a good diagnostic performance, with $\mathrm{SEN}=89.3 \%, \mathrm{SPE}=94.6 \%, \mathrm{ACC}=94.5 \%, \mathrm{LR}+=16.51, \mathrm{LR}-=0.11$, and AUC $=0.93$ (95\% CI 0.91-0.96) (Table 3). The optimal cutoff of G-17 by the maximal Youden index was 14.62 $\mathrm{pmol} / \mathrm{L}$, which was fairly close to the in-house upper limit and displayed similar diagnostic strength (AUC $=0.94$, $95 \%$ CI 0.91-0.97, $\mathrm{p}=0.294)$. Throughout the progression of stomach diseases, the diagnostic strength of serum G-17 for atrophic gastritis showed a downhill trend across symptomatic cancer-free patients (AUC $=0.85$, $\mathrm{p}=0.142)$ and gastric cancer patients $(\mathrm{AUC}=0.70, \mathrm{p}<0.001)$. Nevertheless, in the entire cross-sectional population, the diagnostic strength was still preserved well ( $A U C=0.92, p=0.648)$ (Fig. 4).

\section{Discussion}

In this cross-sectional study, symptomatic patients and gastric cancer patients in addition to healthy population were simultaneously observed. The prevalence of atrophic gastritis in southwest China were estimated as $15.9 / 1,000$ in healthy population, 28.3/1,000 in upper GI symptomatic patients, and 55.7/1,000 in gastric cancer patients, respectively. High level of serum G-17 (>15 pmol/L) was significantly associated with increased risk of atrophic gastritis in healthy population. Throughout the progression of stomach diseases, the diagnostic strength of serum G-17 for atrophic gastritis showed a downhill trend across more advanced situations. In despite of that, serum G-17 displayed a good performance in predicting atrophic gastritis in entire cross-sectional population.

The prevalence of atrophic gastritis was diverse in different populations in the world. In a Russian asymptomatic cohort, the prevalence of atrophic gastritis was much higher as 108/1,000 persons, while the prevalence of 


\begin{tabular}{|c|c|c|c|c|c|}
\hline & $\begin{array}{l}\text { Healthy controls } \\
(\mathrm{n}=9, \mathbf{4 2 5})\end{array}$ & $\begin{array}{l}\text { Symptomatic cancer- } \\
\text { free patients }(n=671)\end{array}$ & $\begin{array}{l}\text { Gastric cancer } \\
\text { patients }(n=305)\end{array}$ & $p_{1}$ & $\mathbf{p}_{2}$ \\
\hline \multicolumn{6}{|l|}{ Sex } \\
\hline Males & $5,087(54.0 \%)$ & $304(45.3 \%)$ & $208(68.2 \%)$ & $<0.001$ & $<0.001$ \\
\hline Females & $4,338(46.0 \%)$ & $367(54.7 \%)$ & $97(31.8 \%)$ & & \\
\hline \multicolumn{6}{|l|}{ Age (year) } \\
\hline$<20$ & $21(0.2 \%)$ & $1(0.2 \%)$ & 0 & $<0.001$ & $<0.001$ \\
\hline $20-39$ & $2,372(25.2 \%)$ & $110(16.4 \%)$ & $27(8.9 \%)$ & & \\
\hline $40-59$ & $5,781(61.3 \%)$ & $371(55.3 \%)$ & $121(39.7 \%)$ & & \\
\hline $60-79$ & $1,210(12.8 \%)$ & $185(27.6 \%)$ & $149(48.9 \%)$ & & \\
\hline $80-$ & $41(0.4 \%)$ & $4(0.6 \%)$ & $8(2.6 \%)$ & & \\
\hline \multicolumn{6}{|l|}{ PG-I (ug/L) } \\
\hline Normal (70-165) & $5,338(56.6 \%)$ & $375(55.9 \%)$ & $142(46.6 \%)$ & $<0.001$ & $<0.001$ \\
\hline Very low $(<20)$ & $99(1.1 \%)$ & $11(1.6 \%)$ & $6(2.0 \%)$ & & \\
\hline Low $(20,<70)$ & $3,657(38.8 \%)$ & $155(23.1 \%)$ & $49(16.1 \%)$ & & \\
\hline $\operatorname{High}(>165)$ & $331(3.5 \%)$ & $130(19.4 \%)$ & $108(35.4 \%)$ & & \\
\hline \multicolumn{6}{|l|}{ PG-II (ug/L) } \\
\hline Normal (3-15) & 7,633 (81.0\%) & $466(69.4 \%)$ & $149(48.8 \%)$ & $<0.001$ & $<0.001$ \\
\hline Low $(<3)$ & $189(2.0 \%)$ & $16(2.4 \%)$ & $2(0.7 \%)$ & & \\
\hline High $(>15)$ & $1,603(17.0 \%)$ & $189(28.2 \%)$ & $154(50.5 \%)$ & & \\
\hline \multicolumn{6}{|l|}{ PG-I/II ratio } \\
\hline Normal (7-20) & 7,247 (76.9\%) & $514(76.6 \%)$ & $168(55.1 \%)$ & 0.028 & $<0.001$ \\
\hline Very low $(<3)$ & $214(2.3 \%)$ & $22(3.3 \%)$ & $23(7.5 \%)$ & & \\
\hline Low $(3,<7)$ & $1,827(19.4 \%)$ & $109(16.2 \%)$ & $104(34.1 \%)$ & & \\
\hline High $(>20)$ & $137(1.5 \%)$ & $26(3.9 \%)$ & $10(3.3 \%)$ & & \\
\hline \multicolumn{6}{|l|}{ G-17 (pmol/L) } \\
\hline Normal (1-15) & $5,885(62.4 \%)$ & $380(56.6 \%)$ & $187(61.3 \%)$ & $<0.001$ & $<0.001$ \\
\hline Low $(<1)$ & $2,904(30.8 \%)$ & $162(24.1 \%)$ & $28(9.2 \%)$ & & \\
\hline $\operatorname{High}(>15,30)$ & $334(3.5 \%)$ & $73(10.9 \%)$ & $49(16.1 \%)$ & & \\
\hline Very high $(>30)$ & $302(3.2 \%)$ & $56(8.4 \%)$ & $41(13.4 \%)$ & & \\
\hline
\end{tabular}

Table 1. Demography and tests of pepsinogens and gastrin-17 at the baseline. Abbreviations: G-17, gastrin-17; PG-I, pepsinogen-I; PG-II, pepsinogen-II. $\mathrm{p}_{1}$, for comparisons between symptomatic cancer-free patients and healthy controls. $\mathrm{p}_{2}$, for comparisons between gastric cancer patients and healthy controls.

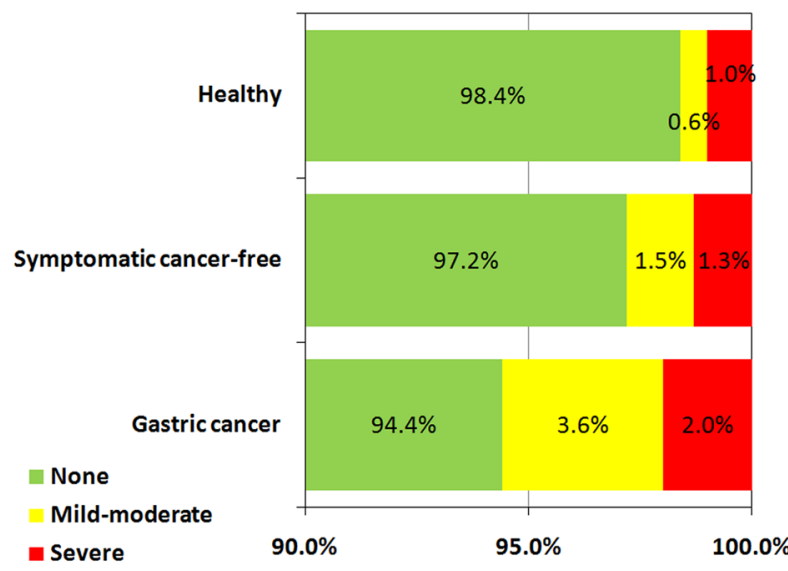

(A)

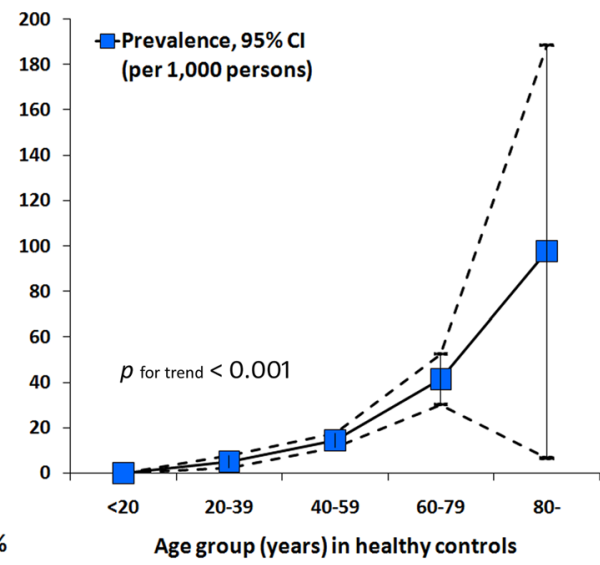

(B)

Figure 2. (A) The proportions of atrophic gastritis by serologic classification in different subjects, and (B) the age-specific prevalence (per 1,000 persons) in healthy controls.

Helicobacter pylori (H. pylori) infection was as high as $76.7 \%$ in the cohort ${ }^{25}$. Similarly, in a Kazakhstan cohort (both asymptomatic and symptomatic), the prevalence of atrophic gastritis was up to 141/1,000 persons, with $\mathrm{H}$. pylori infection infection (but no atrophy) in $62.3 \%$ of the cohort ${ }^{26}$. In a Cameroon small-sized dyspeptic series, the prevalence of atrophic gastritis was $66 / 1,000$ persons, and the rate of $\mathrm{H}$. pylori infection was $79.8 \%{ }^{27}$. In Korea, one of the high-incidence nations of gastric cancer, a multicenter study found the prevalence of endoscopic 


\begin{tabular}{|c|c|c|c|c|c|c|}
\hline \multirow[b]{2}{*}{ Covariate } & \multicolumn{2}{|c|}{ Healthy controls } & \multicolumn{2}{|c|}{ Symptomatic cancer-free } & \multicolumn{2}{|c|}{ Gastric cancer } \\
\hline & \begin{tabular}{|l|} 
CAG \\
prevalence
\end{tabular} & aOR $(95 \% \mathrm{CI})$ & $\begin{array}{l}\text { CAG } \\
\text { prevalence }\end{array}$ & aOR $(95 \% \mathrm{CI})$ & $\begin{array}{l}\text { CAG } \\
\text { prevalence }\end{array}$ & aOR $(95 \% \mathrm{CI})$ \\
\hline \multicolumn{7}{|l|}{ Sex } \\
\hline Female & $17.1 \%$ & ref. & $38.1 \%$ о & ref. & $72.2 \%$ о & ref. \\
\hline Male & $14.9 \%$ & $1.00(0.68-1.49)$ & $16.4 \%$ & $0.44(0.14-1.33)$ & $48.1 \%$ & $0.71(0.25-2.05)$ \\
\hline \multicolumn{7}{|l|}{ Age (yrs) } \\
\hline$<40$ & $5.0 \%$ & ref. & $0 \%$ & - & $0 \%$ & - \\
\hline $40-59$ & $14.5 \%$ & $1.82(0.93-3.58)$ & $32.3 \%$ & ref. & $66.1 \%$ & ref. \\
\hline $60-$ & $43.2 \%$ & $3.01(1.46-6.21)$ & $37.0 \%$ & $1.20(0.42-3.44)$ & $57.3 \%$ & $1.43(0.50-4.10)$ \\
\hline \multicolumn{7}{|c|}{ Serum G-17 } \\
\hline Normal & $1.9 \%$ & ref. & $7.9 \%$ & ref. & $37.4 \%$ o & ref. \\
\hline Low & $1.7 \%$ & $0.96(0.33-2.76)$ & $6.2 \%$ & $0.87(0.09-8.53)$ & $0 \%$ & - \\
\hline High & $38.9 \%$ & $20.67(9.17-46.55)$ & $13.7 \%$ & $1.97(0.20-19.31)$ & $61.2 \%$ & $1.71(0.42-6.98)$ \\
\hline Very high & $400.7 \%$ & $314.41(166.10-595.12)$ & $250.0 \%$ & $42.40(11.63-154.66)$ & $170.7 \%$ & $5.50(1.78-17.02)$ \\
\hline
\end{tabular}

Table 2. The risks of atrophy gastritis in groups of different subjects. Abbreviations: aOR, adjusted odds ratio; CAG, chronic atrophic gastritis; CI, confidence interval; G-17, gastrin-17; ref., reference.

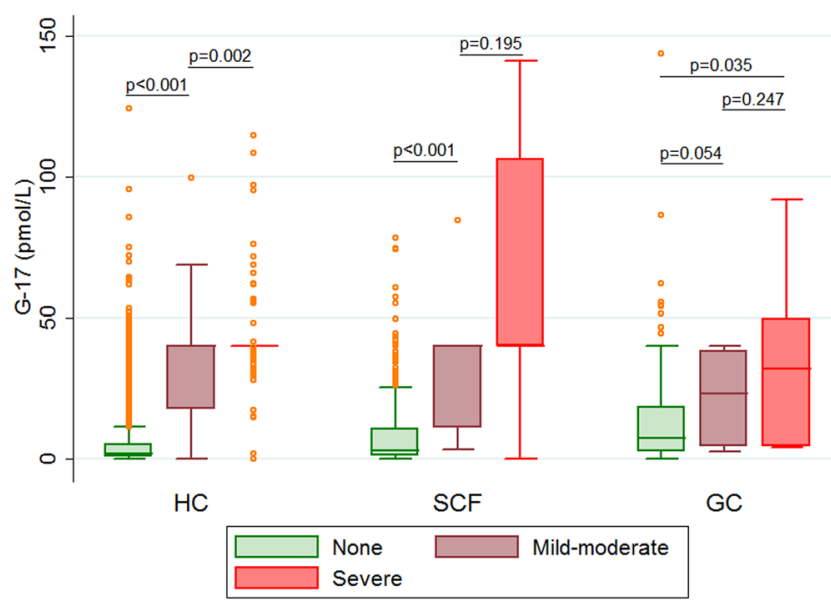

Figure 3. Box plots of serum G-17 levels by severity of atrophic gastritis in healthy controls (HC), symptomatic cancer-free patients (SCF), and gastric cancer patients (GC).

atrophic gastritis was even up to 407/1,000 persons, and H. pylori seropositivity was a risk factor for intestinal metaplasia, a more advanced precancerous lesion ${ }^{28}$. Additionally, a Swedish long-term cross-sectional study revealed the prevalence of atrophic gastritis was associated with the H. pylori seropositivity ${ }^{29}$.

The information on the prevalence of atrophic gastritis was scarce in Chinese. In contrast, the healthy and symptomatic subjects in the SIGES study appeared only $15.9 / 1,000$ and 28.3/1,000 persons had atrophic gastritis. Among the elder healthy persons, the prevalence were $41.3 / 1,000$ and $97.6 / 1,000$ persons in the age groups $60-79$ years and $\geq 80$ years, respectively. The lower prevalence in southwest China may attribute to the decreasing rate of $\mathrm{H}$. pylori infection. Although China was a high-incidence nation of gastric cancer, the prevalence of $\mathrm{H}$. pylori infection displayed a apparent geographic disparity ${ }^{9}$. In a longitudinal observation based on sequential cross-sectional studies in Sichuan, the rate of $\mathrm{H}$. pylori infection declined dramatically from $56.6 \%$ to $41.1 \%$ between 2008 and $2014^{30}$. Therefore, it can be inferred that the efforts in health education, screening and control of H. pylori in Sichuan province during the past decade makes the H. pylori-associated atrophic gastritis decreased. A study in two cities of Fujian province indicated the higher prevalence of $H$. pylori and atrophic gastritis might result in a higher risk condition for developing gastric cancer and a greater population mortality rate $^{31}$. Therefore, these evidence based on Chinese population validated the value of massive screening of atrophic gastritis for the sake of gastric cancer control in China.

In the western populations, it was found the combination of serum PG-I, PG-II, G-17, and H. pylori antibody could obtain acceptable sensitivity and specificity to predict atrophic gastritis ${ }^{32}$. In a multicenter study, the combination had good agreement with endoscopic and biopsy findings in gastric histology of atrophy ${ }^{33}$. However, in contrast, another multicenter prospective study found the combination was not accurate enough to predict atrophic gastritis among dyspeptic patients with sensitivity $50 \%$ and specificity $80 \%{ }^{34}$. Due to inconsistent results, a meta-analysis pooled twenty diagnostic studies on the combination, and found the summarized sensitivity was 74.7\% (95\% CI 62.0\%-84.3\%) and specificity was 95.6\% (95\% CI 92.6\%-97.4\%) for diagnosis of atrophic gastritis $^{35}$. Another meta-analysis found the combination performed even better in the corpus atrophy than in the 


\begin{tabular}{|l|l|l|l|l|l|l|l|}
\hline G-17 cutoffs(pmol/L) & SEN & SPE & ACC & LR+ & LR- & AUC $\mathbf{( 9 5} \% \mathbf{C I})$ & p \\
\hline Healthy & & & & & & $0.93(0.91-0.96)$ & ref. \\
\hline$>1$ & $96.7 \%$ & $31.3 \%$ & $32.3 \%$ & 1.41 & 0.11 & & \\
\hline$>15$ & $89.3 \%$ & $94.6 \%$ & $94.5 \%$ & 16.51 & 0.11 & & \\
\hline$>30$ & $80.7 \%$ & $98.1 \%$ & $97.8 \%$ & 41.34 & 0.20 & & \\
\hline Healthy (continuous variable of G-17) & & & & & & $0.94(0.91-0.97)$ & 0.294 \\
\hline$\geq 14.62$ (Youden max) & $90.7 \%$ & $94.3 \%$ & $94.2 \%$ & 15.87 & 0.10 & & \\
\hline Symptomatic cancer-free & & & & & & $0.85(0.74-0.96)$ & 0.142 \\
\hline$>1$ & $94.7 \%$ & $24.7 \%$ & $26.7 \%$ & 1.26 & 0.21 & & \\
\hline$>15$ & $79.0 \%$ & $82.5 \%$ & $82.4 \%$ & 4.52 & 0.26 & & \\
\hline$>30$ & $73.7 \%$ & $93.6 \%$ & $93.0 \%$ & 11.44 & 0.28 & & \\
\hline Gastric cancer & & & & & & $0.70(0.57-0.82)$ & $<0.001$ \\
\hline$>1$ & $100 \%$ & $9.7 \%$ & $14.8 \%$ & 1.11 & 0 & & \\
\hline$>15$ & $58.8 \%$ & $72.2 \%$ & $71.5 \%$ & 2.12 & 0.57 & & \\
\hline$>30$ & $41.2 \%$ & $88.2 \%$ & $85.6 \%$ & 3.49 & 0.67 & & \\
\hline All observations & & & & & & $0.92(0.89-0.94)$ & 0.648 \\
\hline$>1$ & $96.8 \%$ & $30.2 \%$ & $31.4 \%$ & 1.39 & 0.11 & & \\
\hline$>15$ & $85.5 \%$ & $93.2 \%$ & $93.1 \%$ & 12.55 & 0.16 & & \\
\hline$>30$ & $76.3 \%$ & $97.5 \%$ & $97.1 \%$ & 30.34 & 0.24 & & \\
\hline
\end{tabular}

Table 3. The diagnostic capability of serum G-17 for AG status in different subjects. Abbreviations: ACC, accurancy; AUC, area under the curve; CI, confidence interval; G-17, gastrin-17; LR+, positive likelihood ratio; LR -, negative likelihood ratio; ref., reference; SEN, sensitivity; SPE, specificity.

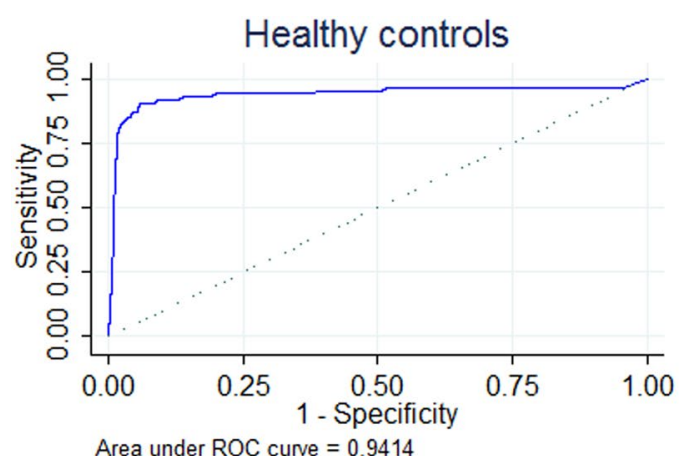

Area under ROC curve $=0.9414$

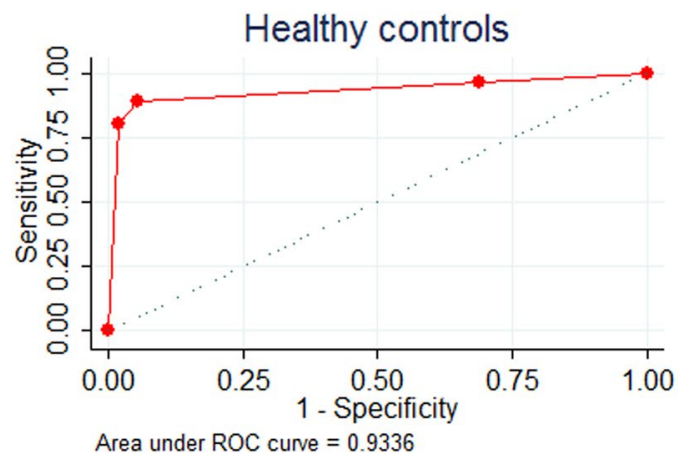

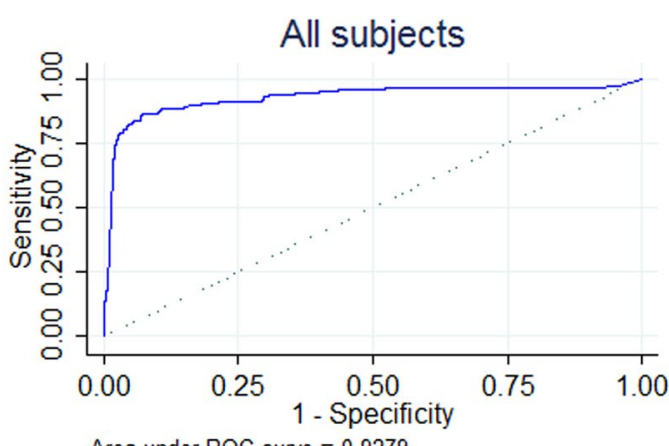

Area under ROC curve $=0.9279$

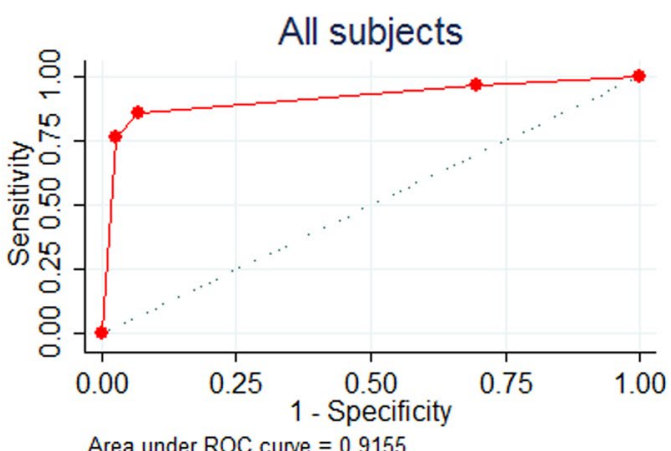

Area under ROC curve $=0.9155$

Figure 4. The ROCs of serum G-17 predicting atrophic gastritis in different subjects. (blue lines for continuous variable; red lines for categorical variable by in-house cutoffs).

antrum atrophy ${ }^{36}$. Likewise, a cross-sectional analysis based on a northeast Chinese population (12,112 subjects) also proved the usefulness of the combination in identifying high-risk individuals for further diagnostic gastroscopy, and stratifying individuals' risk ${ }^{24}$. A recent updated meta-analysis reported the pooled sensitivity was 0.59 (95\% CI 0.38-0.78), while on the other hand, the pooled specificity, diagnostic odds ratio, and AUC were 0.89 (95\% CI 0.70-0.97), 12 (95\% CI 6-25), and 0.81 (95\% CI 0.77-0.84), respectively ${ }^{13}$.

In fact, there was no efficient biomarkers to directly diagnose precancerous lesions of gastric cancer till now $^{37,38}$. However, in the pattern of massive screening, the application of serum pepsinogen assay may be 
reasonable as a primary classification standard. The present study was aimed to validate the predicting strength of serum G-17 in a massive setting. In the future, we plan to validate the combination of both pepsinogens and G-17 to predict atrophic gastritis in a histology-based setting, but in a smaller group of observations. Additionally, the combination of detecting H. pylori, Epstein-Barr virus, and other oncoviruses might be considerable to further investigate the predicting strength of precancerous lesions in a non-invasive pattern ${ }^{30,39-41}$. Therefore, the above findings warrant further investigation based the SIGES population to identify high-risk subpopulation with precancerous condition for endoscopic screening in a cost-effective manner.

Edkins JS first reported gastrin functioning in the chemical mechanism of gastric acid secretion ${ }^{42}$. The expression of gastrin can also be adjusted by the acid feedback ${ }^{43}$. Among the family of gastrin, G-17 particularly plays a critical role in the regulation of gastric acid secretion ${ }^{43}$. Some other researches similarly found high level of serum gastrin was associated with precancerous lesions and gastric cancer in Asians ${ }^{44-47}$. The results of the present study demonstrated that the prevalence of atrophic gastritis tended to increase from benign to malignant lesion. Atrophy might make acid secretion decreased, while the serum G-17 would be increased through acid feedback adjustment. Similarly, the proton pump inhibitor could make the acid secretion inhibited, and led to the elevated level of serum G-17 $7^{48}$. It may be the mechanism why a high level of serum G-17 is potentially able to predict precancerous lesions of gastric cancer.

There were some limitations need consider with caution. First, this was a hospital-based cross-sectional population, rather than a natural population. There might be a certain sampling bias. Second, the classification of atrophic gastritis was dependent on serologic measurement, rather than endoscopy and histology. A minor part of misclassification might not be eliminated in this study. The upper GI endoscopy was not mandatory in the massive health checkup in China, so it was impossible to be improved in a cross-sectional study. The serologic classification by the combination of serum PG-I and PG-I/II ratio could achieve a good diagnostic accurancy, even as high as more than $90 \%{ }^{1,23}$. However, the updated meta-analysis found the pooled sensitivity of serum pepsinogen assay was merely $59 \%$ to predict atrophic gastritis ${ }^{13}$. It means the prevalence of atrophic gastritis might be underestimated in the present study. Third, the health checkup did not involve endoscopic screening and confirmation of the absence of gastric cancer. However, the crude incidence of gastric cancer in China was $33.66 / 100,000$ persons $^{49}$, and the underestimated gastric cancer might be rare in the healthy controls. The sensitivity analysis including the entire cross-sectional population found the contamination with gastric cancer patients $(305 / 10,401)$ did not impair the diagnostic strength of serum G-17 for prewarning of atrophic gastritis. Fourth, there was methodological concerns why low and normal levels of G-17 were combined. The prevalence of atrophic gastritis were quite close between normal and low levels of G-17, while the aORs were not significant in the healthy and symptomatic cancer-free controls, as well as null atrophic gastritis in low level of G-17 in gastric cancer patients (Table 2). Therefore, in the following analysis shown in the Table 3, the serum G-17 was analyzed as a continuous variable rather than a binary variable. Fifth, the administration of the proton pump inhibitor (PPI) was unclear at the baseline, especially in symptomatic and gastric cancer patients. Recent usage of PPI might lead to the elevated level of serum G-1 $7^{48}$. It might be the reason why only very high level ( $\left.>30 \mathrm{pmol} / \mathrm{L}\right)$ of G-17 was associated with atrophic gastritis status in symptomatic and gastric cancer patients. Finally, the consideration of the joint assessment along with $\mathrm{H}$. pylori status in the following investigation of SIGES would be more informative to understand the high-risk subpopulation in southwest China for health policy decision-maker.

In conclusion, population in southwest China had intermediate prevalence of AG, while the prevalence was increased over age or disease progression. In the SIGES study, serum G-17 was found to be a reliable non-invasive measurement to predict AG in Chinese population. However, the decision-making based on serum G-17 in massive screening need further investigate in prospective epidemiologic studies.

Received: 21 June 2019; Accepted: 26 February 2020;

Published online: 11 March 2020

\section{References}

1. Brenner, H., Rothenbacher, D. \& Weck, M. N. Epidemiologic findings on serologically defined chronic atrophic gastritis strongly depend on the choice of the cutoff-value. Int. J. Cancer 121, 2782-2786 (2007).

2. Song, H. et al. Incidence of gastric cancer among patients with gastric precancerous lesions: observational cohort study in a low risk Western population. BMJ. 351, h3867 (2015)

3. Chen, X. Z. et al. Association of helicobacter pylori infection and chronic atrophic gastritis with risk of colonic, pancreatic and gastric cancer: A ten-year follow-up of the ESTHER cohort study. Oncotarget 7, 17182-17193 (2016).

4. Ikeda, F. et al. Combination of Helicobacter pylori Antibody and Serum Pepsinogen as a Good Predictive Tool of Gastric Cancer Incidence: 20-Year Prospective Data From the Hisayama Study. J. Epidemiol. 26, 629-636 (2016).

5. Digestive Endoscopy Group of Chinese Medical Association and Oncologic Endoscopy Committee of Chinese Anti-Cancer Association. China Consensus on Early Gastric Cancer Screening, Endoscopic Diagnosis and Treatment (2014. 4, Changsha). Chin J Gastroenterol. 19, 408-427. (Chinese) (2014).

6. Malfertheiner, P. et al. Management of Helicobacter pylori infection-the Maastricht IV/Florence Consensus Report. Gut 61, 646-664 (2012).

7. Chen, X. Z., Zhang, W. H. \& Hu, J. K. A difficulty in improving population survival outcome of gastric cancer in mainland China: low proportion of early diseases. Med. Oncol. 31, 315 (2014).

8. Chen, X. Z., Hu, J. K., Yang, K., Wang, L. \& Lu, Q. C. Short-term evaluation of laparoscopy-assisted distal gastrectomy for predictive early gastric cancer: a meta-analysis of randomized controlled trials. Surg. Laparosc. Endosc. Percutan Tech. 19, 277-284 (2009).

9. Wang, R. \& Chen, X. Z. High mortality from hepatic, gastric and esophageal cancers in mainland China: 40 years of experience and development. Clin. Res. Hepatol. Gastroenterol. 38, 751-756 (2014).

10. Chen, X. Z., Liu, Y., Wang, R., Zhang, W. H. \& Hu, J. K. Improvement of cancer control in mainland China: epidemiological profiles during the 2004-10 National Cancer Prevention and Control Program. Lancet 388, S40 (2016).

11. Yang, K. et al. Strategies to improve treatment outcome in gastric cancer: a retrospective analysis of patients from two high-volume hospitals in Korea and China. Oncotarget 7, 44660-44675 (2016).

12. Miki, K. \& Urita, Y. Using serum pepsinogens wisely in a clinical practice. J. Dig. Dis. 8, 8-14 (2007). 
13. Bang, C. S., Lee, J. J. \& Baik, G. H. Prediction of Chronic Atrophic Gastritis and Gastric Neoplasms by Serum Pepsinogen Assay: A Systematic Review and Meta-Analysis of Diagnostic Test Accuracy. J. Clin. Med. 8, E657 (2019).

14. Adamu, M. A., Weck, M. N., Rothenbacher, D. \& Brenner, H. Incidence and risk factors for the development of chronic atrophic gastritis: five year follow-up of a population-based cohort study. Int. J. Cancer. 128, 1652-1658 (2011).

15. Wang, X. et al. The correlation between histological gastritis staging- 'OLGA/OLGIM' and serum pepsinogen test in assessment of gastric atrophy/intestinal metaplasia in China. Scand. J. Gastroenterol. 52, 822-827 (2017).

16. Lee, J. Y. et al. Correlations among endoscopic, histologic and serologic diagnoses for the assessment of atrophic gastritis. J. Cancer Prev. 19, 47-55 (2014).

17. Fock, K. M. et al. Asia-Pacific consensus guidelines on gastric cancer prevention. J. Gastroenterol. Hepatol. 23, 351-365 (2008).

18. Rehfeld, J. F. The new biology of gastrointestinal hormones. Physiol. Rev. 78, 1087-1108 (1998).

19. Sipponen, P. et al. Atrophic Gastritis Serum Levels of Amidated Gastrin-17 and Pepsinogen I in Atrophic Gastritis: An Observational Case-Control Study. Scand. J. Gastroenterology. 37, 785-791 (2009).

20. Sun, L. et al. A comprehensive evaluation of fasting serum gastrin-17 as a predictor of diseased stomach in Chinese population. Scand. J. Gastroenterol. 49, 1164-1172 (2014).

21. Rozengurt, E. \& Walsh, J. H. Gastrin, CCK, signaling, and cancer. Annu. Rev. Physiol. 63, 49-76 (2001).

22. Wang, X. et al. The Diagnostic Value of Gastrin-17 Detection in Atrophic Gastritis: A Meta-Analysis. Med. 95, e3599 (2016).

23. Yoshida, T. et al. Cancer development based on chronic active gastritis and resulting gastric atrophy as assessed by serum levels of pepsinogen and Helicobacter pylori antibody titer. Int. J. Cancer. 134, 1445-1457 (2014).

24. Tu, H. et al. A Serological Biopsy Using Five Stomach-Specific Circulating Biomarkers for Gastric Cancer Risk Assessment: A MultiPhase Study. Am. J. Gastroenterol. 112, 704-715 (2017).

25. Roman, L. D. et al. Prevalence of H. pylori Infection and Atrophic Gastritis in a Population-based Screening with Serum Biomarker Panel (GastroPanel(R)) in St. Petersburg. Anticancer. Res. 36, 4129-4138 (2016).

26. Benberin, V. et al. Prevalence of H. pylori infection and atrophic gastritis among symptomatic and dyspeptic adults in Kazakhstan. A hospital-based screening study using a panel of serum biomarkers. Anticancer. Res. 33, 4595-4602 (2013).

27. Ebule, I. A., Longdoh, A. N. \& Paloheimo, I. L. Helicobacter pylori infection and atrophic gastritis. Afr. Health Sci. 13, 112-117 (2013).

28. Joo, Y. E. et al. Prevalence and risk factors of atrophic gastritis and intestinal metaplasia: a nationwide multicenter prospective study in Korea. Gut Liver 7, 303-310 (2013).

29. Song, H. et al. Increase in the Prevalence of Atrophic Gastritis Among Adults Age 35 to 44 Years Old in Northern Sweden Between 1990 and 2009. Clin. Gastroenterol. Hepatol. 13, 1592-1600 e1591 (2015).

30. Wang, R., Zhang, M. G., Chen, X. Z. \& Wu, H. Risk population of Helicobacter pylori infection among Han and Tibetan ethnicities in western China: a cross-sectional, longitudinal epidemiological study. Lancet 388, S17 (2016).

31. Zheng, K. C. et al. Serum pepsinogens, gastrin-17 and Helicobacter pylori antibody in the residents of two cities in china with distinct mortality rates of gastric cancer. Tohoku J. Exp. Med. 228, 289-294 (2012)

32. Sipponen, P., Harkonen, M., Alanko, A. \& Suovaniemi, O. Diagnosis of atrophic gastritis from a serum sample. Clin. Lab. 48, 505-515 (2002).

33. Vaananen, H. et al. Non-endoscopic diagnosis of atrophic gastritis with a blood test. Correlation between gastric histology and serum levels of gastrin-17 and pepsinogen I: a multicentre study. Eur. J. Gastroenterol. Hepatol. 15, 885-891 (2003).

34. McNicholl, A. G. et al. Accuracy of GastroPanel for the diagnosis of atrophic gastritis. Eur. J. Gastroenterol. Hepatol. 26, 941-948 (2014).

35. Zagari, R. M. et al. Systematic review with meta-analysis: diagnostic performance of the combination of pepsinogen, gastrin-17 and anti-Helicobacter pylori antibodies serum assays for the diagnosis of atrophic gastritis. Aliment Pharmacol Ther. (2017).

36. Syrjanen, K. A Panel of Serum Biomarkers (GastroPanel(R)) in Non-invasive Diagnosis of Atrophic Gastritis. Systematic Review and Meta-analysis. Anticancer. Res. 36, 5133-5144 (2016).

37. Chen, X. Z. et al. Correlation between serum CA724 and gastric cancer: multiple analyses based on Chinese population. Mol. Biol. Rep. 39, 9031-9039 (2012).

38. Chen, X. Z. et al. Associations between serum CA724 and HER2 overexpression among stage II-III resectable gastric cancer patients: an observational study. Oncotarget 7, 23647-23657 (2016).

39. Wang, R., Liu, K., Chen, X. Z. \& SIGES research group. Associations between gastric cancer risk and virus infection other than Epstein-Barr virus: The protocol of a systematic review and meta-analysis based on epidemiological studies. Med. 98, el6708 (2019).

40. Chen, X. Z., Chen, H., Castro, F. A., Hu, J. K. \& Brenner, H. Epstein-Barr virus infection and gastric cancer: a systematic review. Med. 94, e792 (2015)

41. Chen, X. Z., Wang, R. \& Hu, J. K. Hepatitis B virus infection and gastric cancer risk: pitfalls in the potential association. Br. J. Cancer. 112, $1844(2015)$

42. Edkins, J. S. The chemical mechanism of gastric secretion. J. Physiol. 34, 133-144 (1906).

43. Smith, A. M. \& Watson, S. A. Review article: gastrin and colorectal cancer. Aliment. Pharmacol. Ther. 14, 1231-1247 (2000).

44. Loong, T. H. et al. Serum pepsinogen and gastrin-17 as potential biomarkers for pre-malignant lesions in the gastric corpus. Biomed. Rep. 7, 460-468 (2017).

45. Wu, K. C. et al. Diagnosis of atrophic body gastritis in Chinese patients by measuring serum pepsinogen. Chin. J. Dig. Dis. 5, 22-27 (2004).

46. Cao, Q., Ran, Z. H. \& Xiao, S. D. Screening of atrophic gastritis and gastric cancer by serum pepsinogen, gastrin-17 and Helicobacter pylori immunoglobulin G antibodies. J. Dig. Dis. 8, 15-22 (2007).

47. Yu, G., Wang, G. X., Wang, H. G., Mo, F. F. \& Tang, B. B. The value of detecting pepsinogen and gastrin-17 levels in serum for precancerous lesion screening in gastric cancer. Neoplasma 66, 637-640 (2019).

48. Agreus, L. et al. Clinical use of proton-pump inhibitors but not $\mathrm{H} 2$-blockers or antacid/alginates raises the serum levels of amidated gastrin-17, pepsinogen I and pepsinogen II in a random adult population. Scand. J. Gastroenterol. 44, 564-570 (2009).

49. Chen, W. et al. Cancer incidence and mortality in China in 2013: an analysis based on urbanization level. Chin. J. Cancer Res. 29, $1-10(2017)$.

\section{Acknowledgements}

This study was conducted from the Sichuan Gastric Cancer Early Detection and Screening (SIGES) project. The 1.3.5 Project for Disciplines of Excellence, West China Hospital, Sichuan University (No. ZY2017304), and Wu Jieping Medical Foundation (No. 320.2710.1815), the funder had no role in study design, data collection and analysis, decision to publish, or preparation of the manuscript. This study was partially represented in the Korea International Gastric Cancer (KINGCA) Week 2019, Incheon, Korea, April 11-13 2019.

\section{Author contributions}

R.W. for data retrieval, statistics and manuscript preparation. X.Z.C. for study registration, application of ethical approval, statistics and manuscript preparation. 


\section{Competing interests}

The authors declare no competing interests.

\section{Additional information}

Supplementary information is available for this paper at https://doi.org/10.1038/s41598-020-61472-7.

Correspondence and requests for materials should be addressed to X.-Z.C.

Reprints and permissions information is available at www.nature.com/reprints.

Publisher's note Springer Nature remains neutral with regard to jurisdictional claims in published maps and institutional affiliations.

(c) (i) Open Access This article is licensed under a Creative Commons Attribution 4.0 International License, which permits use, sharing, adaptation, distribution and reproduction in any medium or format, as long as you give appropriate credit to the original author(s) and the source, provide a link to the Creative Commons license, and indicate if changes were made. The images or other third party material in this article are included in the article's Creative Commons license, unless indicated otherwise in a credit line to the material. If material is not included in the article's Creative Commons license and your intended use is not permitted by statutory regulation or exceeds the permitted use, you will need to obtain permission directly from the copyright holder. To view a copy of this license, visit http://creativecommons.org/licenses/by/4.0/.

(C) The Author(s) 2020 